3 Authors:

4 Katharine Ricke $(a, b)$

5 Laurent Drouet (c)

6 Ken Caldeira (d)

7 Massimo Tavoni (c,e)

9 Affiliations:

10 a School of Global Policy and Strategy, University of California San Diego, La Jolla,

11 California, USA

12 b Scripps Institution of Oceanography, University of California, San Diego, La Jolla,

13 California, USA

14 c RFF-CMCC European Institute on Economics and the Environment (EIEE), Milan, Italy

15 d Carnegie Institution for Science, Stanford, CA, USA

16 e Politecnico di Milano, Department of Management, Economics and Industrial

17 Engineering, Milan, Italy

Abstract/First Paragraph:

20 The social cost of carbon (SCC) is a commonly employed metric of the expected 21 economic damages expected from carbon dioxide $\left(\mathrm{CO}_{2}\right)$ emissions. While useful in 22 an optimal policy context, a world-level approach obscures the heterogeneous 23 geography of climate damages and vast differences in country-level contributions 24 to global SCC, as well as climate and socio-economic uncertainties, which are 25 larger at the regional level. Here we estimate country-level contributions to SCC 26 using recent climate model projections, empirical climate-driven economic damage 27 estimations, and socioeconomic projections. Central specifications show high 28 global SCC values (median: $417 \$ / \mathrm{tCO}, 66 \%$ confidence intervals: $177-805 \$ / \mathrm{tCO}$ ) 29 and country-level SCC which are unequally distributed. However, the relative 30 ranking of countries is robust to different specifications: countries incurring large 31 fractions of the global cost consistently include India, China, Saudi Arabia and the 32 United States. 
34 The social cost of carbon (SCC) represents the economic cost associated with climate 35 damage (or benefit) resulting from the emission of an additional ton of $\mathrm{CO}_{2}$. One way to 36 compute it is by taking the net present value of the difference between climate change 37 damages along with a baseline climate change pathway and the same pathway with an 38 additional incremental pulse release of carbon dioxide. The SCC provides an economic 39 valuation of the marginal impacts of climate change. It has been estimated hundreds of 40 times in the past three decades ${ }^{10}$ using a range of assumptions about uncertain 41 parameters (such as social discount rate, economic growth, and climate sensitivity).

42 Recent estimates ${ }^{1-6}$ of SCC range from approximately $\$ 10 /$ tonne of $\mathrm{CO} 2$ to as much as

$43 \$ 1000 / \mathrm{tCO}$. A recent report issued by the US National Academies highlighted the many 44 challenges and opportunities associated with improving estimates of SCC. ${ }^{11}$

46 Among the state-of-the-art contemporary estimates of SCC are those calculated by the 47 US Environmental Protection Agency (EPA). The latest figures equal to $\$ 12, \$ 42$ and $\$ 62$ 48 per metric tonne of $\mathrm{CO} 2$ emitted in 2020 for 5,3 and 2.5 percent discount rates 49 respectively ${ }^{1}$. These estimates are used, among other purposes, to inform US 50 environmental rulemakings. Various alternative approaches to estimating SCC have been 51 employed over the years, including more sophisticated treatments of time, risk and equity 52 preferences ${ }^{12-17}$, as well as those that incorporate more recent representations of climate 53 damages and feedbacks ${ }^{18-21}$. A recent expert elicitation of climate scientists and 54 economists ${ }^{2}$ found a mean SCC of approximately $\$ 150-200$ per tonne of CO2.

The global SCC captures the externality of $\mathrm{CO} 2$ emissions, and is thus the right value to use from a global welfare perspective. Nonetheless, country level contributions to the SCC are important for various reasons. Mapping domestic impacts can allow quantifying noncooperative behavior, and thus better understand the determinants of international cooperation. The governance of climate agreements ${ }^{22,23}$ is a key issue for climate change.

61 The nationally determined architecture of the Paris climate agreement - and its 62 vulnerability to changing national interests- is one important example. Country level 63 estimates can also allow better understand regional impacts, which are important for 64 adaptation and compensation measure. Finally, higher spatial resolution estimation of 65 climate damage and benefits can impact estimates of net global climate damage ${ }^{24,25}$, and 66 its sensitivity to climate and socio-economic drivers. 
68 Existing studies agree on the significant gap between domestic and global values of the

69 SCC, but provide limited agreement on the distribution of the SCC by region ${ }^{26}$. Due to 70 limitations on the availability of country-level climate and economic inputs, no previous 71 analysis has partitioned global SCC into country-level contributions from each individual 72 nation (CSCC). In this paper, we draw upon recent developments in physical and 73 economic climate science to estimate country-level and aggregate SCC and quantify 74 associated uncertainties. The CSCC captures the amount of marginal damage (or, if 75 negative, the benefit) expected to occur in an individual country as a consequence of an 76 additional $\mathrm{CO}_{2}$ emission. While marginal impacts do not capture all information relevant to 77 climate decision making, the distribution of the CSCC provides useful insights into 78 distributional impacts of climate change and national strategic incentives.

\section{Methodological Approach}

81 Following the recommendations of the recent report by the US National Academies of 82 Science, we execute our calculations of social cost of carbon through a process with four 83 distinct components ${ }^{11}$ : a socioeconomic module wherein the future evolution of the 84 economy, including projected emissions of carbon dioxide, is characterized absent the 85 impact of climate change; a climate module wherein the earth system responds to 86 emissions of carbon dioxide and other anthropogenic forcings; a damages module, 87 wherein the economy's response to changes in the Earth system are quantified; and a 88 discounting module, wherein a time series of future damages is compressed into a single 89 present value. In our analysis, we explore uncertainties associated with each module at 90 the global and country level. We focus only on climate impacts, and do not carry out a full91 fledged cost benefit analysis which would require modeling mitigation costs.

93 We develop a method for calculating social cost of carbon that is oriented towards 94 partitioning and quantifying uncertainties. While it follows the same module structure as 95 the integrated assessment models that have been conventionally used to calculate SCC, 96 rather than building reduced-form models of the climate or economy, we use country-level 97 climate projections taken directly from gridded, ensemble climate model simulation data 98 as well as country-level economic damage relationships taken directly from empirical 99 macroeconomic analyses. Because climate and economic quantities are empirical in this 100 analysis, these uncertainties are probabilistic in our output. Socioeconomic and 
discounting uncertainties are assessed parametrically using five socioeconomic scenarios and twelve discounting schemes.

Socioeconomic module: For the socio-economic projections, we use the shared socioeconomic pathway scenarios (SSPs) ${ }^{9}$. The SSPs provide five different storylines of

106 the future (Supplementary Table S1). We use the GDP and population assumptions of the

107 SSPs as well as subsequent work to estimate the emissions associated with each SSP 108 absent climate mitigation policies ${ }^{27}$.

110 Climate module: We match emissions profiles of the SSPs to those of the Representative 111 Concentration Pathways $\left(\mathrm{RCPs}^{28}\right)$ modeled in the fifth Coupled Model Intercomparison 112 Project (CMIP5) ${ }^{7}$ to estimate baseline warming (see Methods). To estimate the response 113 of the climate system to a pulse release of carbon dioxide, we combine results from CMIP5 114 and a carbon cycle model intercomparison project ${ }^{29}$ (Supplementary Tables S2 and S3). 115 Carbon cycle uncertainty is represented by using the global-scale decay of atmospheric 116 carbon dioxide after a pulse release of $\mathrm{CO}_{2}$ into the present-day atmosphere. Climate 117 system response uncertainty is calculated at the population-weighted country level using 118 gridded output from the CMIP5 abrupt4xco2 experiment in which atmospheric $\mathrm{CO}_{2}$ is 119 instantaneously quadrupled from preindustrial. By convoluting the results from these 120 experiments (as $\mathrm{in}^{30}$, but at the population-weighted country-mean level) we derive a 121 range of country-specific transient warming responses to an incremental emission of $\mathrm{CO}_{2}$.

122 To test the sensitivity of our results to the uncertain feedbacks between economic growth 123 and emissions, we perform the calculations for RCPs 4.5, 6.0 and 8.5 for all SSPs.

Damages module: We convert country-level temperature and precipitation changes into 126 country-level damages using empirical macroeconomic relationships derived by Burke et $127 \mathrm{al}^{8}$ and Dell et $\mathrm{al}^{31}$. Their econometric approaches exploit interannual climate variability in 128 historical observations to estimate the impact of climate on economic growth. Estimating 129 the economic damages associated with a given level of warming is a notoriously 130 challenging problem for which there is no perfect state-of-the-art solution ${ }^{11,32}$. Gross 131 domestic product (GDP) is an informative, but highly imperfect measure of welfare ${ }^{33}$. 132 Among its advantages, an empirical macroeconomic approach: captures interactions and 133 feedbacks among sectors of the economy; captures effects of climate on the economy 134 that have been neglected or are difficult to partition and quantify; has higher geographical 
135 resolution (country-level) than existing alternatives; is empirically validated and has 136 confidence intervals which allow to do uncertainty analysis; and is completely transparent

137 and replicable. Because results are sensitive to the econometric specifications, e.g. 138 whether lags are included to capture long run effects, and countries are distinguished 139 between rich and poor to account for different capability to adapt ${ }^{8}$, we compare all the 140 existing empirical specifications. (See Methods and Supplementary Information)

142 Discounting module: We apply these damage functions to our country-level temperature 143 pulse response, SSP and RCP projections, including associated climate and damage 144 function uncertainty bounds (see Methods and Supplementary Figure S1) and then 145 compress the time series of output into country-level SCC values using discounting. 146 Discounting assumptions have consistently been one of the biggest determinants of 147 differences between estimations of the social cost of carbon ${ }^{13,35}$. While intuitive, the use 148 of a fixed discounting rate is not appropriate, particularly when applied universally to 149 countries with highly disparate growth rates and with significant economic losses due to 150 climate change. We thus use growth adjusted discounting determined by the Ramsey 151 endogenous rule ${ }^{36}$ with a range of values for the elasticity of marginal utility and the pure 152 rate of time preference, but also report fixed discounting results in order to demonstrate 153 the sensitivity of SCC calculations to discounting methods.

155 Global results

156 Global SCC (GSCC) is the sum of country-level SCCs. We calculate CSCC for each set 157 of scenario, parameter and model specification assumptions, establishing an uncertainty 158 range based on a bootstrap resampling method (see Methods and Supplementary 159 Methods) and then aggregate to the global level. The median estimates of the global SCC 160 (Figure 1) are significantly higher than the IAWG estimates, primarily due to the higher 161 damages associated with the empirical macroeconomic production function ${ }^{8}$, though 162 similar SCC have been estimated in the past using other methodologies ${ }^{14,21}$. Under the 163 'middle of the road' socioeconomic scenario (SSP2) and its closest corresponding climate 164 scenario (RCP6.0), and the central specification of BHM damage function (short run, no 165 income differentiation) we estimate a median global SCC of $\$ 417 / \mathrm{tCO} 2$ (rate of time 166 preference $=2 \%$, elasticity of marginal utility $=1.5$ ). 
168 The choice of both socioeconomic and climate scenario has an impact on estimated 169 GSCC (Figure 1 and Supplementary Figure S2). For a given RCP, scenarios with strong 170 economic growth and reduced cross-country inequalities (SSP1 and SSP5) have smaller

171 GSCC than worlds with low productivity and persistent or even increasing global inequality 172 (SSP3 and SSP4). For a given SSP, higher emission scenarios lead to higher global SCC. 173 When using fixed time discounting (Supplementary Figure S2), results are significantly 174 different. In particular, global SCCs are lower across scenarios, and the ranking to SSPS 175 and RCPs is often reversed. This highlights the importance of using the appropriate 176 endogenous discounting rules to capture the feedback of climate on the economy.

178 Figure 1 also shows the sensitivity to the impact function specification. Under most 179 socioeconomic scenarios, global SCC is significantly higher and more uncertain when 180 calculated with a long-run (lagged) damage model specification (BHM-LR). This 181 somewhat counterintuitive result indicates that whether climate's primary impact on the 182 economy is through growth or level effects, the negative cumulative effect of climate 183 change on long-term growth is substantial and robust. The GSCC is always lower using 184 the rich/poor specifications of the damages model with confidence intervals that, in most 185 cases, extend into the negative SCC range. The DJO specification of the economic impact 186 function ${ }^{31}$ yields significantly higher GSCC value.

188 Confidence intervals (66\%) illustrated in Figure 1 emphasize the large degree of empirical 189 uncertainty surrounding SCC estimates, even if scenario and structural uncertainties are 190 disregarded. These stem from both the uncertainties of the climate system response to $191 \mathrm{CO}_{2}$ (climate sensitivity) and uncertainties in economic harm expected from climate 192 change (damage function). The latter are especially significant for the long-run 193 specifications, which by construction have larger confidence intervals. 


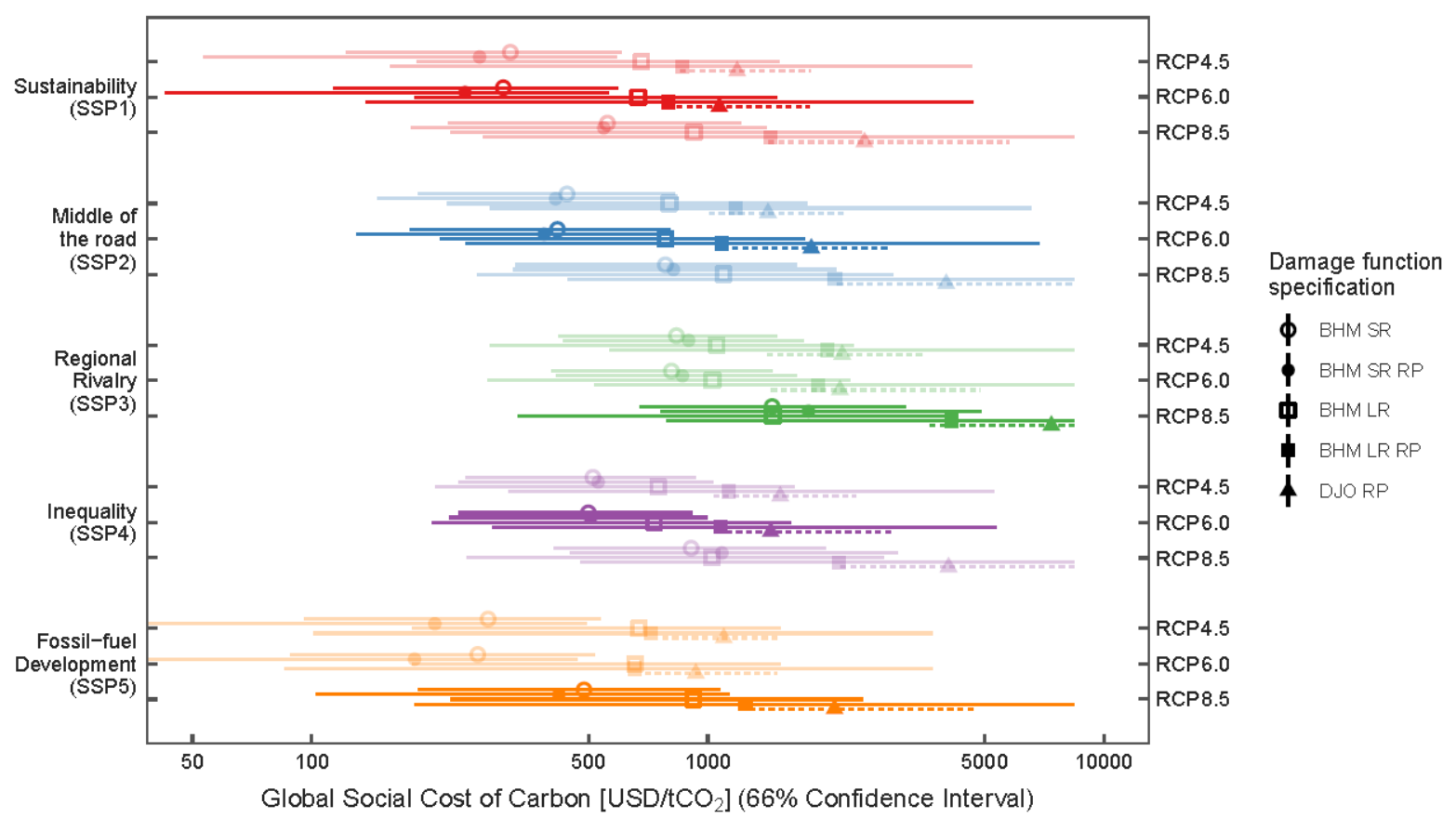
Figure 1 / Global Social Cost of Carbon in 2020 under various assumptions and

200 scenarios. Median estimates and $16.7 \%$ to $83.3 \%$ quantile bounds for global SCC

201 under SSPS 1-5, and RCPS 4.5, 6.0 and 8.5. For each SSP, darker colors indicate the

202 SSP-RCP pairing with superior consistency (see Methods and Supplementary Table

203 S4). Five specifications of damage function: BHM (Short Run, SR, and Long Run, LR;

204 pooled and with Rich and Poor, RP, distinction) and DJO. Values displayed assume

205 growth-adjusted discounting with a pure rate of time preference of $2 \%$ per year and an

206 inter-temporal elasticity of substitution of 1.5. Supplementary Figure S2 shows results

207 with fixed discounting.

208

209 Country-level results

210 These global estimates conceal substantial heterogeneity in country-level contributions to

211 SCC (CSCCs). Figure 2a shows the spatial distribution of CSCCs under a reference

212 scenario (SSP2-RCP6, standard BHM specification). All fixed discounting, alternative

213 scenario, parameterization and specification results are available as a part of the database

214 included in the Supplementary Information.

215

216 India's CSCC is highest (86 [49-157] \$/tCO2; 21\% [20-30\%] of global SCC), followed by 217 the USA (48 \$/tCO2 [1-118]; 11\% [0-15\%] of global SCC) and Saudi Arabia (47 [27-86] $218 \$ / \mathrm{tCO} ; 11 \%$ [11-16\%] of global SCC). Three countries follow at above 20\$/tCO2: Brazil 
219 (24 [14-41] \$/tCO2), China (24 [4-50] \$/tCO2) and United Arab Emirates (24 [14-48] 220 \$/tCO2). Northern Europe, Canada, and the Former Soviet Union have negative CSCC 221 values since their current temperatures are below the economic optimum. These results 222 are among the most sensitive in the analysis, as under the BHM long-run and DJO 223 damage model specifications all countries have positive CSCC. Under the reference case 224 and other short-run model specifications, about $90 \%$ of the world population have a 225 positive CSCC. While the magnitude of CSCC varies considerably depending on scenario 226 and discount rate, the relative distribution is generally robust to these uncertainties. 227 Damage function uncertainty is a larger contributor to overall uncertainty, but at the 228 country level, either climate or damages uncertainty may be larger. The alternative 229 economic damage functions confirms the broad heterogeneity of CSCCs and relative 230 country ranking (see Figure $2 b$ and Supplementary Figure S5).

231
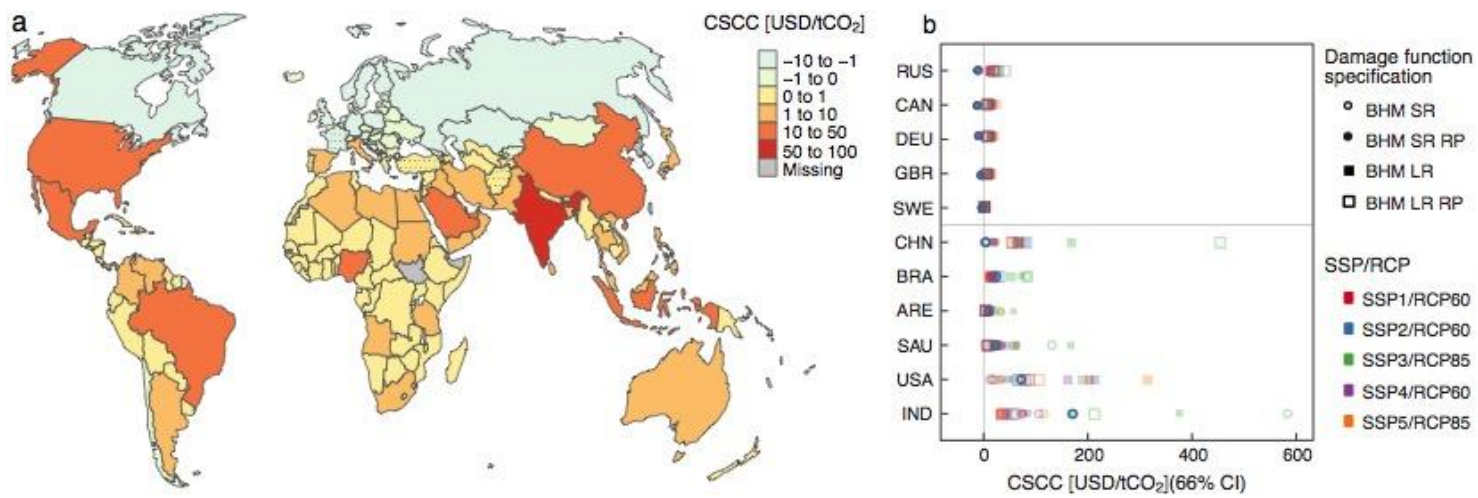

233 Figure 2 / Country-level social costs of carbon (CSCCs). (a) Spatial distribution of

234 median estimates of the CSCC computed for the reference case of scenario

235 SSP2/RCP60, short-run pooled specification of BHM impact function (BHM-SR), and a

236 growth adjusted discount rate with $2 \%$ pure rate of time preference and IES of 1.5.

237 Stippling indicates countries where BHM damage function is not statistically robust ${ }^{8}$ (b)

238 CSCCs for alternative scenarios and damage function specification combinations for the

239 five smallest and six largest CSCCs in the reference case (blue open circles).

241 Consistent with past work on the geography of climate damages ${ }^{4,8,37}$, we find that the 242 international distribution of SCC is inequitable (Lorenz curves in Figure 3). The magnitude 243 of the inequality is sensitive to the model specification of the economic impact function.

244 As discussed above, there is an unsettled debate as to whether empirical evidence points 245 towards the influence of climate on the economy operating primarily via growth or level 
246 effects, something that has been analyzed without definitive conclusion in BHM and follow247 up work ${ }^{38}$. Our results indicate that this uncertainty is consequential from a strategic 248 perspective (i.e., in determining relative gains and losses to particular countries). In 249 particular, with long-run (LR) and DJO specifications all countries have positive CSCCs. 250 This results in higher (almost twice as much) global values of the SCC (as already 251 observed in Figure 1) and lower inequality with respect to the short terms specification. 252 The distinction between income groups in the impact function (rich and poor countries) 253 has smaller impacts, reducing global SCC and either leaving inequality unchanged (for 254 the short-term specification) or lowering it (for the long-term one).
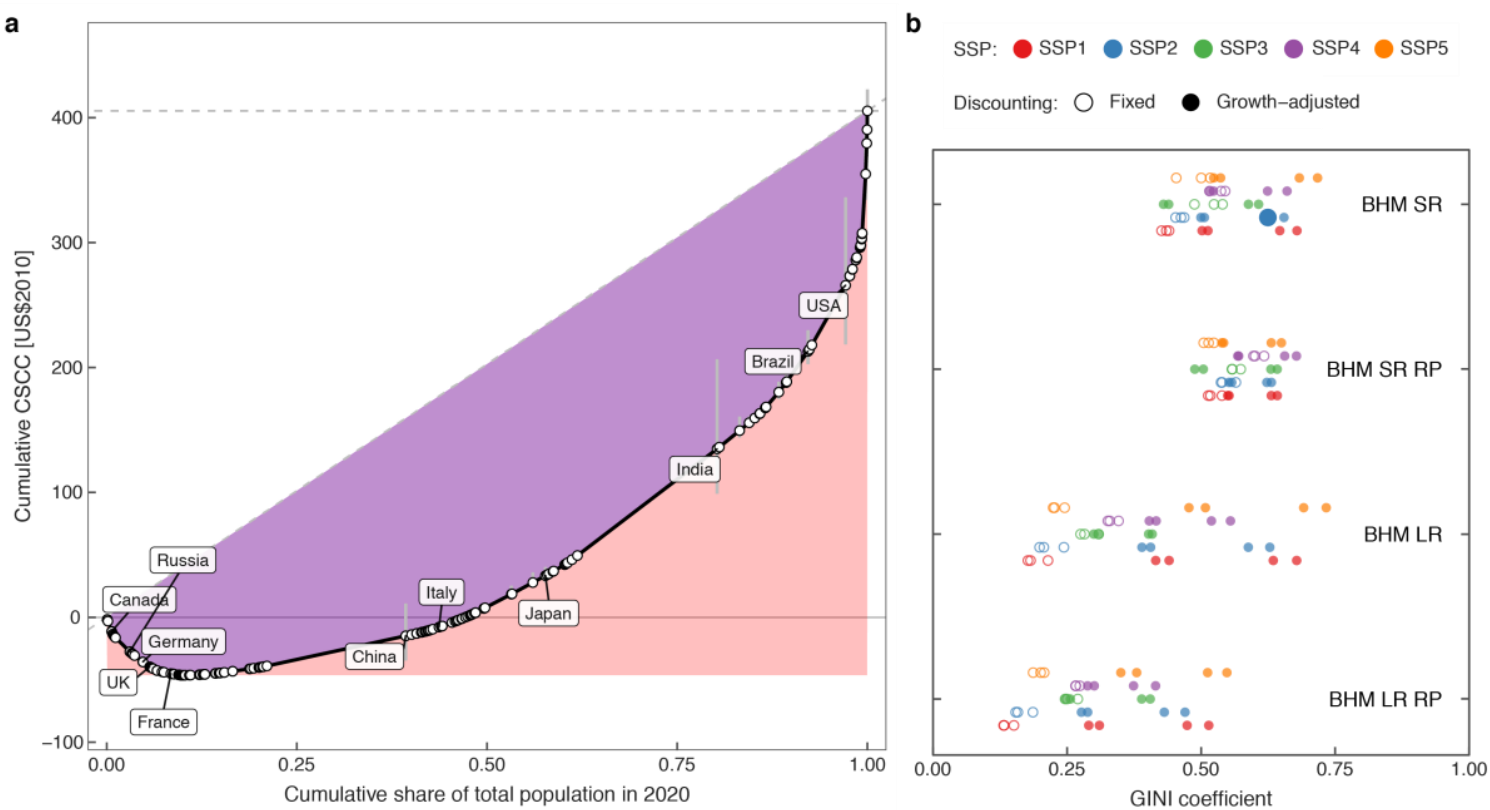

Figure 3 / Lorenz curve and Gini coefficients for the country-level contributions to

258 the Global SCC in 2020. (a) Cumulative global population plotted versus cumulative

259 SCC, with countries ranked by CSCC per capita, produces a Lorenz curve for the

260 reference case of scenario SSP2/RCP60, short-run pooled specification of BHM impact

261 function (BHM-SR), and a growth adjusted discount rate with $2 \%$ pure rate of time

262 preference and IES of 1.5. The red and purple shaded areas illustrate the quantities

263 required to calculate the Gini coefficient, a synthetic metric of heterogeneity/inequality,

264 which is equal to the purple area divided by the sum of the purple and red areas. (b)

265 shows Gini coefficients for all four damage model specifications from top to bottom: the

266 BHM short-run pooled model (SR), short run rich-poor specification (SR-RP), long-run

267 pooled $(L R)$ and the long-run rich-poor (LR-RP). Shared Socioeconomic Pathways 
(SSPS) are distinguished by color for both fixed (open) discounting with rates 2.5\%, 3\% and $5 \%$ and growth-adjusted (solid) discounting with prtp= $(1 \%, 2 \%)$ and ies $=(0.7,1.5)$. The reference case (Gini coefficient=0.62) is illustrated with a large, solid blue point.

272 Figure 3(b) summarizes the inequality of CSCC across all scenarios through Gini 273 coefficients $^{39,40}$ a synthetic measure of global heterogeneity. Under the BHM-SR 274 specification, Gini values increase moderately with the RCP forcing. It is higher for SSP1 275 and SSP5, and significantly lower for SSP3, which is also the socio-economic scenario 276 with the highest global SCC value. Socioeconomic uncertainty also becomes more 277 important to future outcomes under a long-run economic impact models, whereas the rich278 poor distinction plays a smaller role. The discounting method also plays an important role: 279 fixed discounting leads to significantly lower Gini coefficients for CSCC for most 280 specifications.

282 Figure 4 highlights a mapping of winners and losers from climate change among G20 283 nations. While the magnitude of CSCC is subject to considerable uncertainty, the shares 284 of global SCC allocated among world powers remains relatively stable (Supplementary 285 Figures S7-S9) in all short-run impact model specifications. Russia dominates all other 286 nations in gains from emissions, while India is consistently dominated by all other large 287 economies with large losses. Other developing economies, such as Indonesia and Brazil, 288 will accrue a significantly greater share of global SCC than their current share of global 289 emissions. The world's biggest emitters -China and the US- both stand to accrue a smaller 290 share of global SCC than their share of emissions, but are consistently dominated by the 291 EU, Canada, South Korea and -- in the case of the US -- Japan.

293 Relative ranking of SCC is highly consistent among most of the 276 scenario-impact294 discounting uncertainty cases with the notable exception of the relative positions of major 295 world powers occurs under the long-run impact model specifications (Supplementary 296 Figures S7-S9). Countries like Russia, Canada, Germany and France that have negative 297 CSCC under the reference case switch to having among the highest positive CSCCs 298 (Supplementary Figure S9). After the short- and long-run differences, the largest shifts in 299 country-order relative to our reference case occur under the high-emissions SSP5 300 scenario and in the transition between growth-adjusted and fixed discounting 301 (Supplementary Figure S8). 

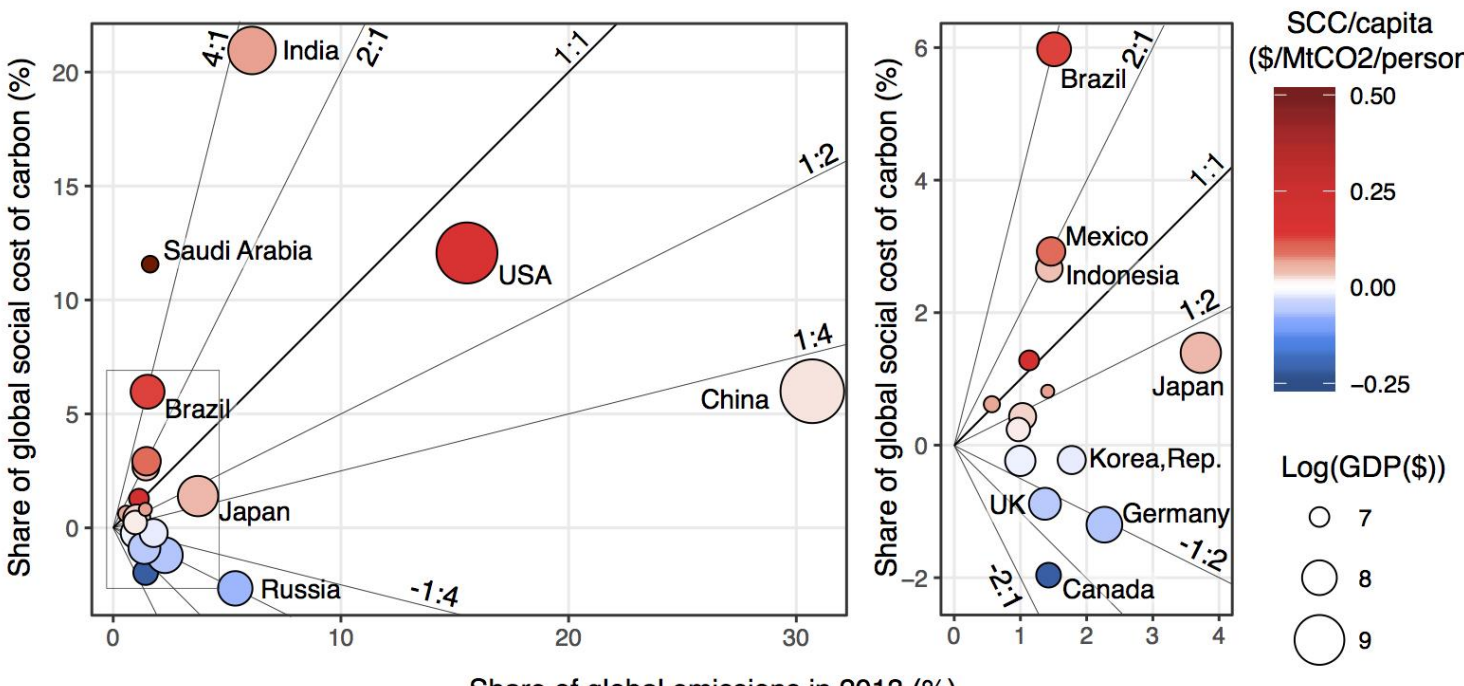

304

Share of global emissions in 2013 (\%)

305 Figure 4 / 'Winners' and 'Losers' of climate change among G20 nations. Country-

306 level shares of global SSC (i.e., CSCC/GSCC) versus shares of 2013 CO2 emissions.

307 CSCC is the median estimate with growth adjusted discounting for SSP2/RCP6.0, BHM-

308 SR reference specification (short run, pooled countries). Bubble size corresponds to the

309 country's GDP (log(USD)) and the color indicates per-capita CSCC (\$/MtCO2/person).

310 Diagonal lines show the ratio of global SCC share to emissions share. Ratios greater

311 than 1:1 indicate that a country's share of global SSC exceeds it share of global

312 emission. Grey box in left panel indicates the bounds of the detail shown in right panel.

\section{Discussion}

315 The discord between country-level shares in $\mathrm{CO}_{2}$ emissions and country-level shares in

316 the social cost of carbon illustrates an important reason why significant challenges persist

317 in reaching a common climate agreement. If countries were to price their own carbon

318 emissions at their own CSCC, approximately only $5 \%$ of the global climate externality

319 would be internalized. At the same time, our results consistently show that the three

320 highest emitting countries (China, the U.S. and India) also have the among the highest

321 country-level economic impacts from a CO2 emission. These high emitter CSCCs are on

322 par with carbon prices foreseen by detailed process IAMs for climate stabilization

323 scenarios (see Supplementary Figure S10). That is, internalizing the domestic SCC in

324 some major emitters could result in emissions pathways for those countries which are 
325 consistent with $1.5-2{ }^{\circ} \mathrm{C}$ temperature pathways. Fully internalizing the $\mathrm{CO} 2$ externality 326 (ie., pricing carbon at global SCC) would allow meeting the Paris Agreement goal and 327 beyond.

329 Empirical, macroeconomic damage functions have advantages and disadvantages 330 compared to the approaches that have typically been used to estimate social cost of 331 carbon in the past. Strengths include transparency, a strong empirical basis and capacity 332 to account for interactions among all sectors of the economy, and for impacts difficult to 333 isolate and quantify. However, there are a number of long-term effects of climate change 334 that are not captured by this type of relationship. We present a number of these excluded 335 contributors in Supplementary Table S5, along with an indication of the likely sign of 336 impacts on CSCCs and global SCC. For example, adjustment costs associated with 337 adaptation are not accounted for in this model. Such costs could be high or, given that 338 climate change is not a surprise, could be modest compared to the type of effects 339 that are represented (and which are demonstrably large). Already in our analysis, impacts 340 from climate change are large enough in some countries to lead to negative discount rates 341 (see Supplementary Figure S11). Most of these additional contributors would be expected 342 to increase the global social cost of carbon.

344 Globalisation and the many avenues by which countries fortunes are linked mean that 345 high CSCC in one place may result in costs as the global climate changes even in places 346 where CSCC is nominally negative. For many countries, the effects of climate change may 347 be felt more greatly through transboundary effects, such as trade disruptions ${ }^{41}$, large-scale 348 migration $^{42}$, or liability exposure ${ }^{43}$ than through local climate damage. While CSCC in 2020 349 is negative for many rich, northern countries, if the non-linear climate damages hold over 350 time, CSCC will become positive in most countries as the planet continues to warm. 351 Furthermore, reducing greenhouse gas emissions can yield positive synergies on other 352 environmental goals, such as improving air quality, which have large welfare impacts 353 already now ${ }^{44}$. These considerations suggest that country-level interests may be more 354 closely aligned to global interests than indicated by contemporary country level 355 contributions to the social cost of carbon. What's more, climate decision making does not 356 occur in a vacuum. Some countries, such as northern Europe and Canada, are leaders 357 on climate policy despite potentially negative SCCs, while other countries with the highest 
358 CSCCs, like USA and India, lag behind. Clearly, a host of other strategic and ethical

359 considerations factor into the international relations of climate change mitigation.

The recent U.S. National Academy of Sciences report on social cost of carbon, the 362 Working Group cites three essential characteristics for future social cost of carbon 363 estimates: scientific basis, uncertainty characterization and transparency ${ }^{11}$. Our work 364 includes improvements upon past estimates of SCC on all three counts. Past estimates 365 of social cost of carbon were based on reduced form climate modules and damage 366 function calibration with limited empirical support ${ }^{45}$, while ours uses output from an 367 ensemble of state-of-the-art coupled climate model simulations and two independently368 generated empirical damage functions. Past estimates of SCC have included limited 369 uncertainty analysis, focusing mostly on a limited set of parameters such as the social 370 discount rate, while our estimates include quantified uncertainty bounds for carbon cycle, 371 climate, economic and demographic uncertainties, while also providing disaggregation to 372 the national level. In addition, past estimates of SCC were often generated using opaque 373 models and/or proprietary software. We provide all of our source code and the full output 374 of our analysis for complete transparency (see Supplementary Data).

376 The high values and profound inequalities highlighted by the country-level estimates of 377 the social costs of carbon provide a further warning of the perils of unilateral or fragmented 378 climate action. We make no claim here regarding the utility of country-level social cost of 379 carbon in setting climate policies. Carbon dioxide emissions are a global externality. 380 Despite "deep uncertainty" ${ }^{46}$ about discounting, socioeconomic pathways and appropriate 381 models of coupling between climate and economy, by all account the estimates of global 382 SCC made by the Interagency Working Group on Social Cost of Greenhouse Gases, 383 United States Government (ref. 1) appear much too low. More research is needed to 384 estimate the geographical diversity of climate change impacts and to help devise policies 385 which align domestic interests to the global good. However, large uncertainties in the 386 precise magnitudes of social cost of carbon, both national and global, cannot overshadow 387 the robust indication that some of the world's largest emitters also have the most to lose 388 from their effects.

\section{Methods}


391 We combine socio-economic, climate and impact data to estimate country-level social

392 costs of carbon, that is the marginal damages from $\mathrm{CO} 2$ emissions, for each of the

393 possible scenarios SSP-RCP, using exogenous and endogenous discounting. Lemoine

394 and Kapnick (2016) uses a similar methodology to calculate growth rate impacts rather

395 than CSCCs based on SSPs and damage estimates in Dell et al (2012). ${ }^{37}$ The

396 sequential process for calculating each CSCC is summarised in Supplementary Figure

397 S1. Global SCC is calculated by summing all CSCCs.

399 Suppl. Table 1 summarises the underlying narratives, which cover different challenges to 400 mitigation and adaptation. Several integrated assessment models have recently

401 completed the implementation of the SSPs, computing for each of them future emissions 402 as well as climate outcomes based on the medium complexity MAGICC6 model. ${ }^{27}$ This 403 allows us to map the SSPs onto four different carbon dioxide emission pathways known 404 as representative concentration pathways (RCPs).

406 Data. The SSP database provides the socio-economic projections at country-level for 407 the 5 SSP narratives (available at https://tntcat.iiasa.ac.at/SspDb ${ }^{32}$ ). The GDP 408 projections were produced by the Organisation for Economic Co-operation and 409 Development (OECD), and the population projections were generated by the 410 International Institute for Applied Systems Analysis (IIASA). We compute annual GDP 411 per capita growth rates for each country. The population-weighted average temperature 412 increase at country-level is calculated for three Representative Concentration Pathways 413 (RCP4.5, RCP6.0 and RCP8.5) using the gridded temperature projections provided by a 414 total of 26 global climate models contributing to the fifth phase of the Coupled Model 415 Intercomparison Project (CMIP5). See Suppl. Table 2. GDP per capita growth rates and 416 temperature increases cover the period 2020-2100. The population-weighted average 417 temperature response over time at country-level to the addition of $1 \mathrm{GtCO} 2$ in the 418 atmosphere is obtained by combining the results from the CMIP5 model's outcomes and 419 a total of 15 carbon-cycle models from a carbon-cycle modelling project ${ }^{30}$ (available at $420 \mathrm{http}: / /$ climatehomes.unibe.ch/ joos/IRF_Intercomparison/). Additionally, baseline 421 temperature at the country-level is computed as the annual population-weighted average 422 temperature increases from 1980 to 2010 from the Willmott and Matsuura gridded 423 observational temperature data set ${ }^{47}$. 
425 Climate projections. Population-weighted country-level temperature time series are

426 calculated for all RCP warming scenarios as well as the abrupt4xco2 experiment.

427 Projections are bias corrected using a 1980-2010 observational baseline ${ }^{47}$. To remove

428 the influence of interannual variability, for the purposes of the SCC calculations, RCP

429 scenario time series represented as a quadratic polynomial fit and abrupt4xco2 time

430 series were represented as a 3-exponential fit. Carbon cycle response to a $\mathrm{CO} 2$ pulse

431 was also represented with a 3-exponential fit.

432

433 Impact projections. We follow the same procedure described in Ref 8 to project the 434 economic impacts from the temperature increase. GDP per capita in country $i$ at year $t$

435 is $G_{i, t}=G_{i, t-1}\left(1+\eta_{i, t}+\delta\left(T_{i, t}\right)\right)$, where $\eta_{\{i, j\}}$ is the growth rate coming from the data, in

436 which no climate change occurs. $\delta\left(T_{i, t}\right)$ is a response function of the temperature

437 increase at year $t$. The projected warming effect is adjusted by the baseline temperature

438 effect (see Ref 8). When applying a BHM rich-poor model, we specify the impact

439 function recursively. Because a number of countries transition from poor to rich within

440 the course of a given century-long simulation, for each year simulated, if a country is

441 "rich" the rich-country impact function is applied and if it is "poor" the poor-country impact

442 function is applied. For more details about the application of the alternative climate

443 impact functions, see the Supplementary Information.

445 The Country-level Social Cost of Carbon. The difference in GDP per capita, including 446 the temperature change impacts, between the scenario with and without pulse provide

447 the yearly compound of the CSCC until 2100 (see Supplementary Figure S12). After

4482100 , the compound is kept constant to its value in 2100 until 2200 (or set to zero, see

449 sensitivity analysis in Supp. Table S6). The CSCC is the net present value of the yearly

450 compound multiplied by the population projection.

451

452 Discounting.

453 CSCCs were calculated using both exogenous and endogenous ${ }^{12}$ discounting. For 454 conventional exogenous discounting, two discount rates were used: 3 and 5\%. Results 455 under endogenous discounting were calculated using two rates of pure time preference $456(\rho=1,2 \%)$ and two values of elasticity of marginal utility of consumption $(\eta=0.7,1.5)$ for 457 four endogenous discounting parameterizations. 
460 Recent work (Ref. 28) calculated the forcing paths associated with SSPs by 5 marker

461 models. For each SSP, we consider the RCP forcing scenario with the minimum

462 Euclidian distance between the SSP as a reference scenario (Supplementary Figure

463 S13 and Supplementary Table S4).

\section{Uncertainty.}

466 The uncertainty analysis uses a full ensemble of carbon and climate model combinations 467 to represent climate uncertainty (210-345 model combinations, varying according to the 468 scenarios). Damage function uncertainty is analysed via bootstrapping $(1,000$ sets of 469 parameter values). The combined uncertainty is obtained by convolution. At the end, a 470 Bayesian bootstrap resampling analysis is conducted to provide the estimates of the median and the quantiles along with their confidence interval.

\section{Lorenz curves and Gini coefficients}

474 Lorenz curves are generated using the classical approach ${ }^{39}$. The Gini coefficients are 475 generated using the method of Raffinetti et al $(2015)^{40}$ which developed a coherent 476 approach to incorporating negative income into measurement of inequality, adhering to 477 the principle that 0 designates perfect equality and 1 maximum inequality.

\section{Code and data availability}

480 All scripts used to calculate CSCCs and global SCC are available as a part of the 481 Supplementary Materials. The database of country-level SCCs with uncertainty bounds 482 under all scenarios, model specifications and discounting schemes is available as a part 483 of the Supplementary Materials.

\section{References}

486 1. IAWG, U. Technical support document: Technical update of the social cost of carbon 487 for regulatory impact analysis under executive order 12866. Interag. Work. Group 488 Soc. Cost Carbon U. S. Gov. Wash. DC (2013).

489 2. Pindyck, R. S. The Social Cost of Carbon Revisited. (National Bureau of Economic $490 \quad$ Research, 2016). 
3. Anthoff, D. \& Tol, R. S. J. The uncertainty about the social cost of carbon: A

492 decomposition analysis using fund. Clim. Change 117, 515-530 (2013).

493 4. Moore, F. C. \& Diaz, D. B. Temperature impacts on economic growth warrant

494 stringent mitigation policy. Nat. Clim. Change 5, 127-131 (2015).

495 5. Nordhaus, W. Estimates of the Social Cost of Carbon: Concepts and Results from the 496 DICE-2013R Model and Alternative Approaches. J. Assoc. Environ. Resour. Econ. 1, $497 \quad 273-312$ (2014).

498 6. Bansal, R., Kiku, D. \& Ochoa, M. Price of Long-Run Temperature Shifts in Capital

499 Markets. (National Bureau of Economic Research, 2016).

500 7. Taylor, K. E., Stouffer, R. J. \& Meehl, G. A. An Overview of CMIP5 and the

501 Experiment Design. Bull. Am. Meteorol. Soc. 93, 485-498 (2012).

502 8. Burke, M., Hsiang, S. M. \& Miguel, E. Global non-linear effect of temperature on 503 economic production. Nature 527, 235-239 (2015).

504 9. O'Neill, B. C. et al. A new scenario framework for climate change research: the 505 concept of shared socioeconomic pathways. Clim. Change 122, 387-400 (2013).

506 10. Tol, R. S. J. The Social Cost of Carbon. Annu. Rev. Resour. Econ. 3, 419-443 507 (2011).

508 11. National Academies of Sciences, E. Valuing Climate Damages: Updating 509 Estimation of the Social Cost of Carbon Dioxide. (2017). doi:10.17226/24651

510 12. Anthoff, D., Tol, R. S. J. \& Yohe, G. W. Risk aversion, time preference, and the 511 social cost of carbon. Environ. Res. Lett. 4, 024002 (2009).

512 13. Weitzman, M. L. Tail-Hedge Discounting and the Social Cost of Carbon. J. Econ. $513 \quad$ Lit. 51, 873-882 (2013).

514 14. Ackerman, F. \& Stanton, E. A. Climate Risks and Carbon Prices: Revising the 515 Social Cost of Carbon. Econ. Open-Access Open-Assess. E-J. 6, 1 (2012). 
516 15. Hope, C. Discount rates, equity weights and the social cost of carbon. Energy

517 Econ. 30, 1011-1019 (2008).

518 16. Cai, Y., Judd, K. L. \& Lontzek, T. S. The Social Cost of Carbon with Economic 519 and Climate Risks. ArXiv150406909 Q-Fin (2015).

520 17. Adler, M. et al. Priority for the worse-off and the social cost of carbon. Nat. Clim.

$521 \quad$ Change 7, 443-449 (2017).

522 18. Moyer, E., Woolley, M., Glotter, M. \& Weisbach, D. Climate Impacts on Economic

523 Growth as Drivers of Uncertainty in the Social Cost of Carbon. (2013).

524 19. Kopp, R. E., Golub, A., Keohane, N. O. \& Onda, C. The Influence of the

525 Specification of Climate Change Damages on the Social Cost of Carbon. (Social

526 Science Research Network, 2012).

527 20. Nordhaus, W. Estimates of the Social Cost of Carbon: Concepts and Results

528 from the DICE-2013R Model and Alternative Approaches. J. Assoc. Environ. Resour.

529 Econ. 1, 273-312 (2014).

530 21. Cai, Y., Judd, K. L. \& Lontzek, T. S. The Social Cost of Stochastic and

531 Irreversible Climate Change. (National Bureau of Economic Research, 2013).

532 22. Barrett, S. Self-Enforcing International Environmental Agreements. Oxf. Econ.

533 Pap. 46, 878-894 (1994).

534 23. Carraro, C. \& Siniscalco, D. Strategies for the international protection of the 535 environment. J. Public Econ. 52, 309-328 (1993).

536 24. Adams, R. M., McCarl, B. A. \& Mearns, L. O. The Effects of Spatial Scale of 537 Climate Scenarios on Economic Assessments: An Example from U.S. Agriculture. in 538 Issues in the Impacts of Climate Variability and Change on Agriculture (ed. Mearns, L.

$539 \quad$ O.) $131-148$ (Springer Netherlands, 2003). doi:10.1007/978-94-017-1984-1_6

540 25. Pizer, W. et al. Using and improving the social cost of carbon. Science 346, $541 \quad 1189-1190(2014)$. 
542 26. Nordhaus, W. D. Revisiting the social cost of carbon. Proc. Natl. Acad. Sci.

$543201609244(2017)$.

544 27. Riahi, K. et al. The Shared Socioeconomic Pathways and their energy, land use,

545 and greenhouse gas emissions implications: An overview. Glob. Environ. Change

546 doi:10.1016/j.gloenvcha.2016.05.009

547 28. Moss, R. H. et al. The next generation of scenarios for climate change research

548 and assessment. Nature 463, 747-756 (2010).

549 29. Joos, F. et al. Carbon dioxide and climate impulse response functions for the 550 computation of greenhouse gas metrics: a multi-model analysis. Atmos Chem Phys

$551 \quad 13,2793-2825(2013)$.

552 30. Ricke, K. L. \& Caldeira, K. Maximum warming occurs about one decade after a 553 carbon dioxide emission. Environ. Res. Lett. 9, 124002 (2014).

554 31. Dell, M., Jones, B. F. \& Olken, B. A. Temperature Shocks and Economic Growth:

555 Evidence from the Last Half Century. Am. Econ. J. Macroecon. 4, 66-95 (2012).

556 32. Diaz, D. \& Moore, F. Quantifying the economic risks of climate change. Nat.

$557 \quad$ Clim. Change 7, 774 (2017).

558 33. Jones, C. I. \& Klenow, P. J. Beyond GDP? Welfare across Countries and Time. $559 \quad$ Am. Econ. Rev. 106, 2426-2457 (2016).

560 34. Blanc, E. \& Schlenker, W. The Use of Panel Models in Assessments of Climate 561 Impacts on Agriculture. Rev. Environ. Econ. Policy 11, 258-279 (2017).

562 35. Guo, J., Hepburn, C., Tol, R. S. J. \& Anthoff, D. Discounting and the social cost 563 of carbon: a closer look at uncertainty. Environ. Sci. Policy 9, 216, 205 (2006).

564 36. Ramsey, F. P. A Mathematical Theory of Saving. Econ. J. 38, 543-559 (1928).

565 37. Lemoine, D. \& Kapnick, S. A top-down approach to projecting market impacts of 566 climate change. Nat. Clim. Change 6, 51-55 (2016). 
567 38. Burke, M., Davis, W. M. \& Diffenbaugh, N. S. Large potential reduction in 568 economic damages under UN mitigation targets. Nature 557, 549-553 (2018).

569 39. Gastwirth, J. L. The Estimation of the Lorenz Curve and Gini Index. Rev. Econ.

$570 \quad$ Stat. 54, 306-316 (1972).

571 40. Raffinetti, E., Siletti, E. \& Vernizzi, A. On the Gini coefficient normalization when

572 attributes with negative values are considered. Stat. Methods Appl. 24, 507-521

573 (2015).

574 41. Oh, C. H. \& Reuveny, R. Climatic natural disasters, political risk, and

575 international trade. Glob. Environ. Change 20, 243-254 (2010).

576 42. Bohra-Mishra, P., Oppenheimer, M. \& Hsiang, S. M. Nonlinear permanent

577 migration response to climatic variations but minimal response to disasters. Proc.

$578 \quad$ Natl. Acad. Sci. 111, 9780-9785 (2014).

579 43. Thornton, J. \& Covington, H. Climate change before the court. Nat. Geosci. 9, 3$580 \quad 5(2016)$.

581 44. Rao, S. et al. A multi-model assessment of the co-benefits of climate mitigation 582 for global air quality. Environ. Res. Lett. 11, 124013 (2016).

583 45. Pindyck, R. S. Climate Change Policy: What Do the Models Tell Us? J. Econ. Lit. $584 \quad 51,860-872(2013)$.

585 46. Lempert, R. J. Shaping the next one hundred years: new methods for 586 quantitative, long-term policy analysis. (Rand Corporation, 2003).

587 47. Matsuura, K. \& Willmott, C. Terrestrial Air Temperature and Precipitation: 19005882006 Gridded Monthly Time Series, Version 1.01. Univ. Del. Httpclimate Geog Udel $589 \quad$ Educlimate (2007).

590

591 Supplementary Information is available in the online version of the paper. 592 
593 Acknowledgments M.T. would like to thank Marshall Burke for an early discussion of

594 these ideas and about the climate impact functions. K.R. thanks Craig Mclntosh and

595 Juan Moreno-Cruz for helpful discussions during the revisions of this manuscript. M.T.

596 received funding from the European Research Council under the European Union's

597 Seventh Framework Programme (FP7/2007-2013) / ERC grant agreement n 336155 -

598 project COBHAM. L.D received funding from the EU's Horizon 2020 research and

599 innovation programme under grant agreement 642147 (CD-LINKS).

600

601 Author Contributions M.T. conceived of the study. K.R. performed the climate data

602 analysis. L.D. replicated the economic damage functions and performed the CSCC

603 calculations and uncertainty analysis. K.R., M.T. and L.D. analyzed the results. K.R. and

604 M.T. wrote the manuscript. All authors discussed the results and provided input on the

605 manuscript.

606

607 Author Information Reprints and permissions information is available at

608 www.nature.com/reprints. The authors declare no competing financial interests.

609 Correspondence and requests for materials should be addressed to K.R.

610 (kricke@ucsd.edu).

611

612 